\title{
Sclerotherapy with Povidone lodine Instillation into the Renal Pelvis in the Management of Non-Parasitic Chyluria: Our Experience
}

\author{
Mohammad Abdul Aziz, ${ }^{1}$ Shafiqur Rahman ${ }^{2}$, ATM Mowladad Chowdhury ${ }^{3}$, Mirza Mahbubul Hasan ${ }^{4}$ \\ Nurun Nahar Happy ${ }^{5}$, Tasneem Mahjabeen ${ }^{6}$
}

\begin{abstract}
Background \& objective: To evaluate the effectiveness and safety of $0.2 \%$ povidone iodine renal pelvic instillation as minimally invasive therapy for chyluria resistant to conservative treatment.

Materials \& Methods: From July 2015 to December 2016, 9 patients with chyluria were treated. There were 2 males and 7 females ranging from 30-65 years age. Cystoscopic localization of chylous efflux was done. On a day care basis under local anesthesia a $5 \mathrm{Fr}$ open-ended ureteric catheter was introduced on the affected side. Freshly prepared $10 \mathrm{ml} 0.2 \%$ Povidone iodine solution was instilled as a sclerosing agent in the renal pelvis. A total of 3 doses were given at 8-hour intervals. Unilateral instillation was done in 8 cases, and bilateral instillation was done in 1 patient.
\end{abstract}

Results: Eight of 9 patients showed complete clearance. In 1 patient, recurrence was noted and a repeat injection was given after 4 weeks, with success.

Conclusion: Our experience shows that povidone iodine is a safe and effective sclerosing agent in the management of chyluria.

Key words: Sclerotherapy, Povidone iodine, Renal pelvis, Non-parasitic chyluria.

\section{Introduction:}

Chyluria, the passage of chyle in the urine, is due to the rupture of retroperitoneal lymphatics into the pyelocaliceal system, giving urine a milky appearance. This communication is caused by the obstruction of lymphatic drainage proximal to intestinal lacteals, resulting in dilatation of distal lymphatics and the eventual rupture of lymphatic vessels into the urinary collecting system. ${ }^{1}$ Although the condition is not life-threatening, it, if left untreated, leads to significant morbidity because of hematochyluria, recurrent colicky attacks, nutritional problems due to heavy protein losses and immunosupression resulting from lymphocyturia. ${ }^{2}$

Chyluria is a condition with spontaneous remissions and exacerbation. Most patients are treated conservatively, which includes a high-protein and low-fat diet with medium-chain triglycerides. ${ }^{2}$ Anti-filarial drugs are not helpful in this late manifestation of parasitic infestation by Wuchereria

\section{Author's information:}

${ }^{1}$ Dr. Mohammad Abdul Aziz, Department of Urology, Bangladesh Institute of Research \& Rehabilitation in Diabetes, Endocrine and Metabolic Disorders (BIRDEM), Dhaka

${ }^{2}$ Dr. Shafiqur Rahman, Department of Urology, Bangladesh Institute of Research \& Rehabilitation in Diabetes, Endocrine and Metabolic Disorders (BIRDEM), Dhaka

${ }^{3}$ Dr. ATM Mowladad Chowdhury, Department of Urology, Bangladesh Institute of Research \& Rehabilitation in Diabetes, Endocrine and Metabolic Disorders (BIRDEM), Dhaka

${ }^{4}$ Dr. Mirza Mahbubul Hasan, Department of Urology, Bangladesh Institute of Research \& Rehabilitation in Diabetes, Endocrine and Metabolic Disorders (BIRDEM), Dhaka

${ }^{5}$ Dr. Nurun Nahar Happy, Department of Plastic Surgery and 100 Bed Burn Unit, Dhaka Medical College \& Hospital, Dhaka

${ }^{6}$ Dr. Tasneem Mahjabeen, Department of Dermatology, Bangladesh Institute of Research \& Rehabilitation in Diabetes, Endocrine and Metabolic Disorders (BIRDEM), Dhaka

Correspondence: Dr. Mohammad Abdul Aziz, Cell Phone:+880 1712154513. Email: shafiqur04@gmail.com 
bancrofti. ${ }^{3}$ Renal pelvic instillation sclerotherapy (RPIS) using various sclerosants in different concentrations is often used to cause sclerosis of pyelolymphatic fistulae in those not responding to conservative treatment. Povidone iodine as a sclerosing agent was used successfully in a few studies. ${ }^{4-6}$ We report our experience with the use of povidone iodine as a sclerosing agent in the management of chyluria in 9 patients.

\section{Materials \& Methods:}

From July 2015 to December 2016, nine patients (ranging from 30-65 years) presenting with chyluria confirmed by ether test, estimation of urinary triglyceride and microscopic visualization of lymphocytes in urinary sediments (lymphocyturia) were consecutively included in the study. Of them 2 were males and 7 females. Chyluria was graded by the severity of the symptoms; patients passing milky-white urine were designated grade I, with milky white urine associated with whitish clots or episodes of clot retention as grade II, and with haematochyluria as grade III. Patients having had previous renal pelvic instillation of sclerotherapy (RPIS) and presenting later with chyluria from the same renal unit were designated 'recurrent'. After a detailed clinical evaluation, patients were investigated for haemoglobin, total and differential leukocyte counts, serum proteins and serum creatinine and blood urea nitrogen. Ultrasonography of kidney, ureter and bladder region and IVU were used to exclude other urological diseases. Written informed consent was obtained before starting the therapy. Patients were assessed by cystoscopy under local anesthesia after a fatty meal $(100 \mathrm{~g}$ butter with bread) the night before, to locate the side of the chylous efflux. A $5 \mathrm{Fr}$ ureteric catheter was passed up to the renal pelvis of the affected side and its position confirmed under fluoroscopy. Retrograde pyelography was used to delineate pyelolymphatic fistulae and to assess the pelvic volume. Ten milliliters of sclerosant solution was prepared by mixing $2 \mathrm{ml}$ of $5 \%$ povidone iodine and $8 \mathrm{ml}$ of distilled water. With the patient in the Trendelenburg position, sclerosant solution was given in 8-hour intervals for 3 doses through a ureteric catheter, with the distal end of the ureteric catheter clamped for 5 min after each instillation.
For patients who presented with bilateral reflux, initially one side was treated with the contra-lateral side treated after an interval of 1 week. The patients were followed at 6 weeks and thereafter at 3-monthly intervals by clinical history and urinary chyle examination. Serum creatinine, ultrasonography and/or IVU were used after 6-9 months to detect any harmful effect of RPIS on the kidney.

Results:

Eight of the nine patients showed complete clearance of chyluria. In one patient, recurrence was noted after 1 month and a repeat injection was given for complete clearance. No major complications were seen. One patient developed transient hematuria in the immediate postoperative period which subsided with conservative management. One patient developed acute pyelonephritis, but improved after conservative treatment. No deterioration in renal function was seen in any of the bilateral instillation cases.

\section{Discussion:}

Chyluria is characterized by unpredictable natural history of exacerbation and remissions. The majority of the cases present with passage of milky-white urine along with clots. Other presenting symptoms are dysuria, hematuria, renal colic, backaches and urinary tract infections. ${ }^{7}$ Chyluria usually does not affect the general health. In its severe form, the disease causes significant weight loss, cachexia, malnutrition, hypoproteinemia and immunosuppression. ${ }^{8}$ It is seen most commonly in endemic areas such as Southeast Asia, China, Japan, tropical sub-Saharan Africa and South America. ${ }^{9-10}$ Wucheraria bancrofti is considered to be the parasitic cause for chyluria in the endemic areas. Although rare, non-parasitic causes of chyluria include tuberculosis, congenital anomalies, trauma, post-surgery infections and malignancy. ${ }^{11}$ Goel and associates had reported that ether test failed to confirm chyluria in $73(69 \%)$ of 106 patients in whom it was later confirmed by the presence of lymphocytes in urinary sediments. Therefore, lymphocyturia is a more sensitive tool to confirm the diagnosis of chyluria than the ether test. ${ }^{5}$ Postprandial urinary lipids, especially urinary triglycerides, are reliable marker in the evaluation of 
chyluria. ${ }^{12}$ Urinary triglycerides were routinely done in all our patients with test positivity in all cases.

Additional investigations such as hemoglobin, total and differential leukocyte count, blood urea, serum creatinine and serum proteins, urine culture and sensitivity, ICT for filarial and urine for acid-fast bacilli have to be done. Ultrasonography also has to be done to rule out any other urological causes. Intravenous urography is not routinely recommended. Lymphangiography is the procedure of choice for identification of fistulous communication. It typically shows marked dilation and tortuosity of the lymphatics around the hilar regions of kidney with opacification of the collecting system. Because of its invasiveness and the technical skill required in performing this procedure, it is not currently used as a diagnostic tool. Furthermore, complications such as local tissue necrosis, fat embolism, hypersensitivity reaction and exacerbation of lymph edema have been reported. ${ }^{13,14}$

A noninvasive method of lymphoscintigraphy using 99mTc-nanocolloid has been reported with equal accuracy. ${ }^{15}$ CT and MRI have also been useful in making the diagnosis. CT shows dilated channels resembling a mass of confluent low-density lymph nodes. MR urography shows mesh work of multiple tubular tortuous fluid filled structures in the retroperitoneum. Further evaluation is done by cystoscopy and retrograde pyelography after a heavy fat meal to confirm the site and side of the lymphaticourinary fistula. ${ }^{16}$

Chyluria is not a life-threatening disease. The treatment is safe, minimally invasive and at the same time effective also. RPIS is widely being used because it is minimally invasive and effective.

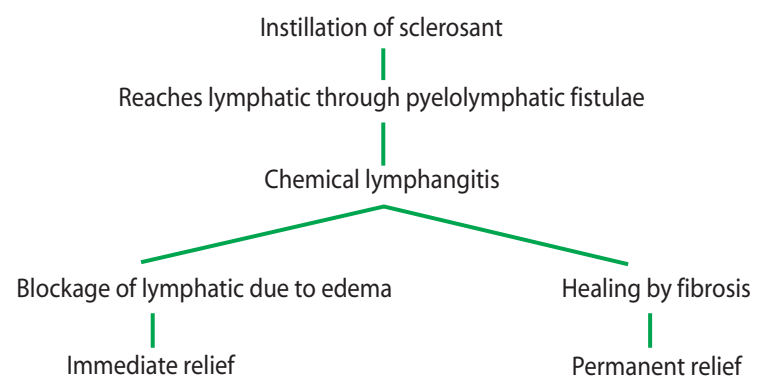

Figure 1: Sclerotherapy-Mechanism of Action
Injected sclerosant induces inflammatory reactions after reaching lymphatics through the pyelolymphatic fistula. This leads to chemical lymphangitis and edema of the lymphatic channels, and resultant blockade leads to immediate relief. Finally, healing by fibrosis causes permanent remission. ${ }^{17}$

Various sclerosant agents have been described: silver nitrate $1-3 \%$, sodium iodide $1-25 \%$, potassium bromide $10-25 \%$, dextrose 50\%, urograffin $76 \%$, and hypertonic saline. ${ }^{18}$ In the past, silver nitrate as a sclerosant was commonly used in concentration ranging from $0.1-3 \% .{ }^{19-20}$ Although it was effective in $80 \%$ of the cases, it was not without complications. The solution is insoluble, susceptible to light and needs to be freshly prepared and autoclaved before use..$^{21-22}$

Shanmugam et al. ${ }^{4}$ used a single instillation of povidone iodine in 5 patients. All his patients were free of symptoms at the 6-month follow-up. Goel et al. ${ }^{5}$ performed instillation of $0.2 \%$ povidone iodine at 8-hour intervals for 3 days. They noted recurrence in $22 \%$ of the patients and concluded that povidone iodine was as effective as silver nitrate. A combination of $5 \mathrm{ml}$ of povidone iodine with $5 \mathrm{ml}$ of $50 \%$ dextrose has been used with complete clearance in $87 \%$ of the patients. ${ }^{23}$

In our study, we used $2 \mathrm{ml}$ of $5 \%$ povidone iodine dissolved in $8 \mathrm{ml}$ of distilled water instilled every $8 \mathrm{~h}$ into the renal pelvis for 3 doses. In 8 of 9 patients $(89 \%)$, complete clearance was noted. One patient required repeat injection. One patient was lost to follow-up. One patient developed hematuria in the immediate postoperative period and 1 patient developed acute pyelonephritis. The overall success rate in our procedure was $100 \%$.

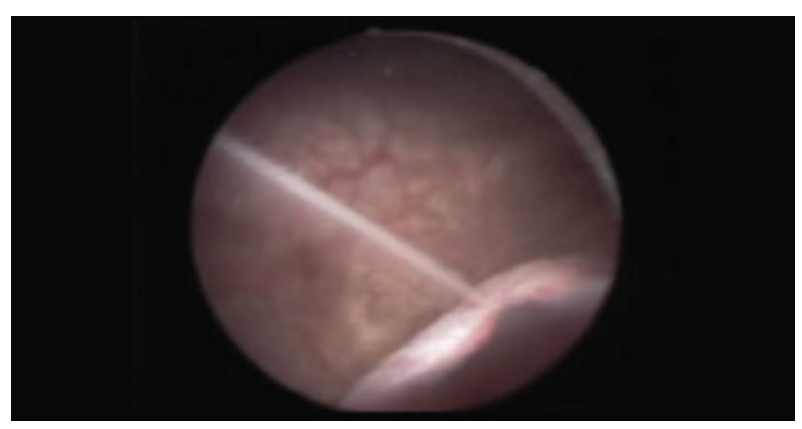

Figure 2: Chyluria: left side efflux during cystoscopy 


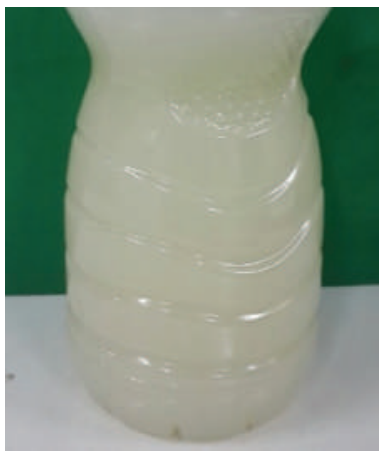

Figure 3: Pre-procedure urine sample

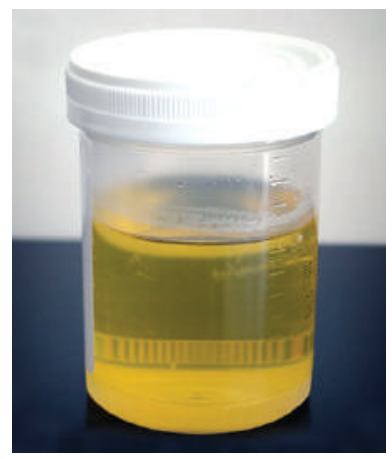

Figure 4: Urine sample 2 weeks after sclerotherapy

\section{Conclusion:}

The study concluded that instillation of $0.2 \%$ povidone iodine as a sclerosant agent in the renal pelvis of patients of chyluria is safe, effective and associated with minimal morbidity. However, randomized control studies will confirm its efficacy better. In addition, split function test before and after the therapy would better clarify the impact of this regimen on renal function.

\section{References:}

1. Hemal AK, Gupta NP: Retroperitoneoscopic lymphatic management of intractable chyluria. J Urol 2002;167:2473-76.

2. Singh I, Dargan P, Sharma N. Chyluria - a clinical and diagnostic stepladder algorithm with review of literature. Indian J Urol 2004;20:79-85.

3. Ansari MS. Medical treatment of filariasis and chyluria. Indian J Urol 2005;21:24-26.

4. Shanmugan TV, Prakash JV, Sivashankar G. Povidone Iodine used as a sclerosing agent in the treatment of chyluria. Br J Urol 1998;82:587.

5. Goel S, Mandhani A, Srivastava A, Kapoor R, Gogoi S, Kumar $A$, et al. Is povidone iodine an alternative to silver nitrate for renal pelvic instillation chemotherapy in chyluria? BJU Int 2004;94:1082-85.

6. Nandy PR, Dwivedi US, Vyas N, Prasad M, Dutta B, Singh PB. Povidone iodine and dextrose solution combination sclerotherapy in chyluria. Urology 2004;64:1107-09.

7. WHO Expert Committee on Filariasis. Lymphatic filariasis: the disease and its control: fifth report of the WHO Expert Committee on Filariasis. WHO Tech Rep Ser 1992;821:1-71.

8. Buck AA. Filariasis; in Strickland GT (ed): Hunters Tropical Medicine, ed 7. Philadelphia, WB Saunders, 2002, p. 713-72.
9. Dalela D. Issues in etiology and diagnosis making of chyluria. Indian J Urol 2005;21:18-23.

10. Cheng JT, Mohan S, Nasir SH, D'Agati VD. Chyluria presenting as milky urine and nephrotic-range proteinuria. Kidney Int 2006;70:1518-22.

11. Yamauchi S. Chyluria: clinical, laboratory and statistical study of 45 personal cases observed in Hawaii. J Urol $1945 ; 54: 318-47$.

12. Koo CG, Van Langenberg A. Chyluria. A clinical study. J $R$ CollSurg Edinb 1969;14:31-41.

13. Peng HW, Chou CF, Shiao MS, Lin E, Zheng H-J, Chen CC, Fan PC. Urine lipids in patients with a history of filariasis. Urol Res 1997;25(3):217-21.

14. Henry JB, Lauzon RB, Schumann GB. Basic examination of urine; in Henry JB (ed): Clinical Diagnosis and Management by Laboratory Methods. Philadelphia, WB Saunders, 1996, p. 411-56.

15. Kittredge RD, Hashim S, Roholt HB, Van Itallie TB, Finby $N$. Demonstration of lymphatic abnormalities in a patient with chyluria. Am J Roentgenol Radium Ther Nucl Med 1963;90:159-65.

16. Choi JK, Weidmer HS. Chyluria: lymphangiographic study and review of literature. J Urol 1964;92:723-27.

17. Zaman MU, Hussain RP, Khan KA, Khan G, Ahmad MN. 99mTc-nanocolloid localization of lymphorenal fistula causing chyluria. J Coll Physicians Surg Pak 2008; 18:515-16.

18. Govil S, Jusutus A, Lakshminarayan R, Nayak S, Devasia A, Gopalkrishnan G. Retroperitoneal lymphatics on CT and MR. Abdom Imaging 2007;32(1):53-55.

19. Gyanendra S, Vinayak C, Rajgopal K, Anshu S, Bari DB. Fluoroscopy guided instillation therapy in chyluria using combination of povidone iodine with contrast agent: is a single instillation sufficient? Int Braz J Urol 2008; 34:270-76.

20. Singh KJ, Srivastava A. Nonsurgical management of chyluria (sclerotherapy). Indian J Urol 2005;21:55-58.

21. Dalela D, Rastogi M, Goel A, Gupta VP, Shankhwar SN. Silver nitrate sclerotherapy for 'clinically significant' chyluria: a prospective evaluation of duration of therapy. Urol Int 2004;72(4):335-40.

22. Sabnis RB, Punekar SV, Desai RM, Bradoo AM, Bapat SD. Instillation of silver nitrate in treatment of chyluria. Br J Urol 1992;70:660-62.

23. Dash SC, Bhargav Y, Saxena S, Agarwal SK, Tiwari SC, Dinda A. Acute renal failure and renal papillary necrosis following instillation of silver nitrate for treatment of chyluria. Nephrol Dial Transplant 1996;11:1841-42. 